\title{
Awareness of hepatic arterial variants is required in surgical oncology decision making strategy: Case report and review of literature
}

\author{
ROBERT SITARZ $^{1,2^{*}}$, MONIKA BERBECKA $^{2^{*}}$, JERZY MIELKO $^{1}$, KAROL RAWICZ-PRUSZYŃSKI $^{1}$, \\ GRZEGORZ STAŚKIEWICZ ${ }^{2,3}$, RYSZARD MACIEJEWSKI ${ }^{2}$ and WOJCIECH POLKOWSKI ${ }^{1}$
}

Departments of ${ }^{1}$ Surgical Oncology, ${ }^{2}$ Human Anatomy and ${ }^{3}$ Radiology, Medical University of Lublin, 20-081 Lublin, Poland

Received September 28, 2017; Accepted January 22, 2018

DOI: $10.3892 / \mathrm{ol} .2018 .8106$

\begin{abstract}
Surgery for the treatment of pancreatic cancer remains the gold standard, however, the identification of the vascular supply of the pancreas and the nearby organs remains a crucial difficulties in a curative resection. During pancreatic head resection for carcinoma dissection of regional arterial vasculature is mandatory. Normal coeliac and hepatic arterial anatomy occurs in $\sim 50-70 \%$ of patients and multiple variations have been described. Knowledge of multiple arterial anomalies is essential in hepato-pancreatico-billary surgery to avoid unnecessary complications. The present study presents coeliac trunk and common hepatic artery (CHA) anomalies along with their clinical importance, as reviewed according to the available literature. Patients diagnosed with cancer of the pancreatic head were hospitalized for staging and planning of radical surgical therapy. Computed tomography (CT) revealed a large tumour mass in the head of the pancreas and CHA, which branched directly from the superior mesenteric artery. A three-dimensional CT reconstruction revealed a demonstrative vascular anomaly, which was confirmed during an operation. Despite the anomalous origin of the CHA, pylorus preserving pancreatoduodenectomy and regional lymph node
\end{abstract}

Correspondence to: Dr Robert Sitarz, Department of Surgical Oncology, Medical University of Lublin, 11 Staszica Street, 20-081 Lublin, Poland

E-mail: r.sitarz@umlub.pl

*Contributed equally

Abbreviations: CT, computer tomography; CAT, celiac artery trunk; CHA, common hepatic artery; ERCP, cholangiopancreatography; GDA, gastroduodenal artery; HAS, hepatic arterial system; HPB, hepato-pancreatico-billary; LGA, left gastric artery; LHA, left hepatic artery; SA, splenic artery; SMA, superior mesenteric artery; PHA, propia hepatic artery; RHA, right hepatic artery

Key words: vascular anomaly, hepatomesenteric trunk, incomplete truncus coeliacus, computer tomography scan, perioperative staging, pancreatoduodenectomy dissection without intraoperative complications was performed in each case. The patient's postoperative clinical course was uneventful and adjuvant chemotherapy could be administered without delay. In the multidisciplinary treatment of pancreatic carcinoma the surgeon and radiologist must be aware of the aberrant anatomy in order to avoid potential complications. As CT scans used for the preoperative staging are of diagnostic value for vascular anomaly, it is required for appropriate surgical decision making.

\section{Introduction}

The increase in the hepatobiliary and pancreatic cancer surgery, the anatomic variations of the celiac axis (also called the celiac trunk or celiac artery (CAT) in the literature) and the hepatic arteries is of paramount importance in hepato-pancreatico-billary (HPB) surgery. In the literature vascular anomalies in the peripancreatic region are divided into variations of the superior mesenteric area, the celiac trunk and hepatic artery. The information about the abdomen arterial anatomy is derived from the radiology and anatomy literature. Typically, coeliac trunk arises anteriorly from abdominal aorta at the level of twelfth thoracic vertebrae, just the aorta enters the abdomen. Then courses anteriorly or slightly anterolateral in the lesser sac and at the upper border of the pancreas divides into three branches: Left gastric artery (LGA), splenic artery (SA) and common hepatic artery (CHA) (1). A normal CAT anatomy was found in $89.1 \%$ of patients (1). The length of coeliac axis from its origin to the place where it gave of main branches is $1.5-2 \mathrm{~cm}$. The diameters of SA, CHA and LGA are 5,6 and $4 \mathrm{~mm}$, respectively (2).

The patterns of hepatic arterial anatomy are not constant. The normal anatomy of the hepatic artery is a CHA arising from the coeliac axis and coursing to the point where the gastroduodenal artery (GDA) arises, beyond which it becomes the proper hepatic artery (PHA). CHA typically passes forward for a short distance in the retroperitoneum and then emerges at the superior border of the pancreas and left side of common hepatic duct. After arising from CAT, CHA turns upwards and runs lateral and adjacent to the common bile duct. GDA is the first branch of CHA which supplies the proximal duodenum and pancreas. The right gastric artery 
takes off shortly thereafter and continues within the lesser omentum along the lesser curve of the stomach. At this point, CHA is referred to as PHA, which courses towards the hilum and soon divides into LHA and RHA. In $80 \%$ cases, RHA courses posterior to the common hepatic duct before entering the hepatic parenchyma; in $20 \%$ cases RHA may lie anterior to the common hepatic duct (3). This normal anatomy of CHA accounting for 25 to $75 \%$ of the cases $(1,4,5)$. Anomalies during embryogenesis however might result in a variety of anatomic variants and most common variants are: From aorta: $0.5-2 \%$; from SMA: $1.5-3.5 \%(1,4,5)$. On the basis of the literature two main paths of CHA can be distinguished. These two variation can have a significant impact on surgical cut: i) The extra-parynchemal path (outside the pancreas head)-CHA is coming out SMA and is passing to the liver on the posterior surface of the pancreas head; in this case the dissection of this artery from the pancreas without pancreas head injury is not difficult; ii) the intra-parynchemal path (in the pancreas head)-CHA is coming out superior mesenteric artery and is going to the liver hilum through head parenchyma; in this case it might be difficult to save intra-parenchymal part of the CHA (when saving of CHA is impossible it is necessary to reconstruct it due to performing end to end anastomosis with gastro-duodenal artery).

We report two cases of associated CAT and CHA anomaly with their clinical importance. This paper is a retrospectively prepared analysis and review of the literature based on two independent rare cases connected with pancreatico-billary surgery. The presented subject is highly in the area of interests of the Department of Surgical Oncology, Medical University of Lublin which mainly concentrate on surgery of gastrointestinal tract cancers; moreover the Department is an academic centre and is focused on training in surgical oncology. We describe hepatic arterial anatomic variants: Where a hepatic arterial system (HAS) arising directly from SMA and traveling posterior to the pancreatic head and vena porta. We discuss the importance of these arterial variants and implications of surgery management.

\section{Case reports}

Case 1. (CHA originated from SMA, hepatomesenteric trunk). A 44-year-old man presented with tumour of the pancreatic head [diameter $1.4 \times 1.1 \mathrm{~cm}$ on computed tomography (CT) scan]. Endoscopic retrograde cholangiopancreatography (ERCP) revealed a common bile duct structure and atypical cells, and a stent was placed. CT scan demonstrated a CHA arising directly from the SMA (Fig. 1). Intraoperatively, a palpable mass $3 \times 4 \mathrm{~cm}$ in the pancreatic head was found and suspected anomalies of the hepatic artery were confirmed after extended Kocher's manoeuvre and careful dissection of peripancreatic and retroperitoneal space with so-called 'artery first' approach. 'Radical' pylorus preserving pancreatoduodenectomy with curative intent was performed. No postoperative complications were observed and patient was discharged from the hospital on 10th postoperative day. Pathologic evaluation revealed a T4N0M0 pancreatic tubular adenocarcinoma resected with positive retroperitoneal margin (R1). Regional lymphadenectomy enabled pathological examintion of 27 lymph nodes without metastases. On 19th postoperative day the patient was readmitted to the hospital due to pancreatic fistula type B. The complication was effectively managed conservatively for 16 days. Thirty five days after operation patient was scheduled for systemic adjuvant chemotherapy.

Case 2. (CHA originated from SMA, hepatomesenteric trunk). A 67-year-old man presented with a pancreatic head cancer. CT scan demonstrated focal tumour in the head of the pancreas. CT arteriography showed a CHA originating from the SMA and traveling posterior to the head of the pancreas as well as posterior to Vena Porta (Fig. 2). After division of the pancreatic neck anterior to the superior mesenteric vein, mobilization of the pancreas head and uncinated process exposed the CHA and GDA. We performed pylorus preserving pancreatoduodenectomy sp. Traverso-Longmire and cholecystectomy. Patient's postoperative hospitalization lasted 10 days. He did well postoperatively and pathologic evaluation confirmed adenocarcinoma tubulare et papillare partim gelatinosum (G1), pT2N1M0 with negative margins. Next pathologist examined 44 lymph nodes and in 3 found component of cancer cells. Patient was qualified for systemic chemotherapy.

\section{Discussion}

Nowadays, there are many improvements and developments in abdomen surgical techniques: upper abdominal videolaparoscopic surgeries, liver transplantation and radiological procedures $(6,7)$. All of invasive procedures in the abdomen need for professional and wide knowledge of the anatomy of the CAT, HAS and their main variations. The frequency of inadvertent or iatrogenic hepatic vascular injury increases in the event of aberrant anatomy and variations. The knowledge of anatomical liver vascular variants is crucial for decreasing operative and postoperative morbidity and mortality during the performance of hepatic and pancreatic surgeries (7-9). Fortunately, along with the development of surgical techniques come the improvement in radiological visualization. Pre-operative imaging can detect even up to $60-80 \%$ of all artery anomalies (8). The gold standard for arterial supply visualization remains angiography, nevertheless the huge impact of multidetector computer tomography (CT) angiography and modern reconstruction programs should be noted. Usage of maximum intensity projection and three-dimensional volume rendering techniques allows the non-invasive visualization of small arteries in multidetector CT angiography (10).

Arterial vascularisation of the gastrointestinal tract is provided by anterior branches at three different levels of the abdominal aorta (coeliac trunk, superior and inferior mesenteric arteries). Haller (1756) was described CAT as the trifurcation which originates on the LGA, SA and CHA $(6,11)$. Since that observation many variations and anomalous were described (7). The normal anatomy of CAT and it branches is observed in $60-89,1 \%$ of cases, while normal hepatic arterial supply can be seen in $52-80.3 \%$ of cases (10). Most of anatomical variations come from foetal developmental changes in the ventral segmental arteries. Tandler (1904) gave an embryological explanation for anatomical variations of CAT (11) where explained that the ventral branches develop initially from the abdominal aorta as paired vessels, which form four roots connected by ventral 


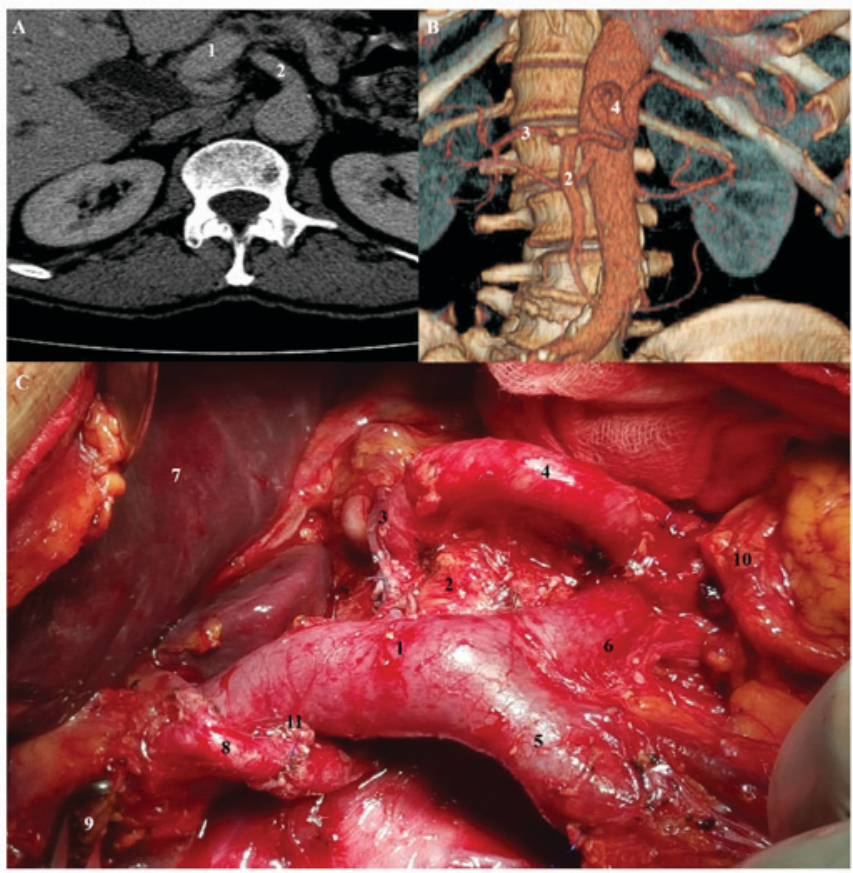

Figure 1. Anomalous origin of common hepatic artery from SMA. (A) Axial scan, abdominal computed tomography, shows proximal common hepatic artery running posterior to portal vein. (B) Volume rendering, abdominal computer tomography. (C) Intraoperative view: 1, portal vein; 2, SMA; 3 , common hepatic artery; 4 , splenic artey; 5 , superior mesenteric vein; 6 , splenic vein; 7 , liver; 8 , proper hepatic artery; 9 , bile ducts; 10 , tail of pancreas; 11, gastroduodenal artery stump. SMA, superior mesenteric artery.

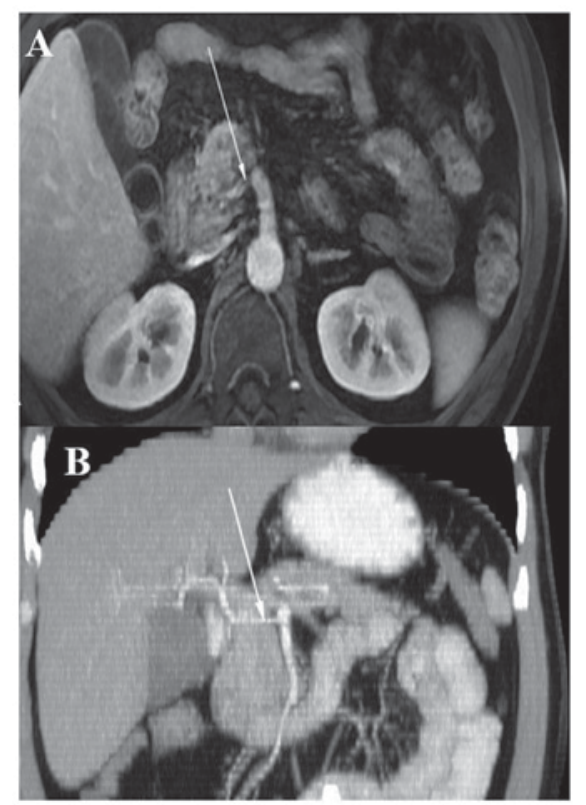

Figure 2. Anomalous origin of common hepatic artery (arrow) from the superior mesenteric artery. (A) Axial scan, magnetic resonance imaging (B) Coronal MIP reformation, computer tomography.

longitudinal anastomosis. LGA is usually formed by the first root, the second root gives the beginning for the SA and the third root creates CHA; SMA develops from the last root, which migrates caudally with the gut $(6,11)$. Quoting Morita, the disappearance of primitive ventral splanchnic arteries and longitudinal anastomosis is the reason for numerous anomalous of the CAT, as it was schematically presented in Fig. 3 (11).

In the paper we aimed to illustrate several types of anatomical variations of celiac trunk, hepatic artery and its main branches, based on description of rare clinical cases. We described two independent cases of such rare anomalies. Moreover, we also focused on surgical implications and an establishment of practical tips for surgeons during abdominal surgery especially HPB surgery.

In the literature classical course of CAT is reported with a frequency of $72-90 \%(6,12)$. According to the Uflacker's classification $(6,13)$ the most commonly observed CAT variations are: Hepatosplenic trunk (3\% of cases), splenogastric trunk $(4 \%)$, hepatogastric trunk $(1 \%)$, hepatomesenteric trunk $(<1 \%)$, the absence of CAT is the most rare $(0,1-4,0 \%)(6,10)$. Among vessel anomalities 'accessory' and 'replaced' vassels can also be qualified, which examples are replaced right hepatic artery (11-21\% cases) and replaced left hepatic artery (3,8-10\%) (8). In the literature coeliac trunk bifurcation is reported at a rate of about $12 \%$ (12).

The mostly chosen classifications for description of anatomical findings and possible surgical implications of CAT variation are presented in Table I. Regarding liver arterial supply, it is described as 'normal anatomy' when the CHA originates the PHA after the emergence of the GDA; next, the PHA separates into right and left hepatic arteries within the hepatoduodenal ligament. The knowledge of hepatic artery supply is essential to avoid iatrogenic complications during HPB surgery; in the presence of anatomical variations accidental ligation, provoking hepatic necrosis, ischemic biliary injury and anastomotic fistula can complicate the peri- and postoperative course (14). With the grooving number of liver transplantation, the importance of the hepatic artery anatomy become crucial and many authors proposed classifications describing liver vascular variations based on their studies (7). The most often described hepatic artery anatomical variations are: i) An anomalous RHA from the SMA (10-21\%); ii) displaced LHA from the LGA (4-10\%); iii) displaced RHA and LHA; iv) an accessory RHA and/or LHA (1-8\%); v) displaced CHA from SMA or aorta $(0,4-4,5 \%)$; or vi) quadrifurcation of hepatic artery (14). In our study we described two independent cases of hepatic arterial anatomic variants where a HAS arised directly from SMA and traveling posterior to the pancreatic head and vena porta. It is named as hepatomesenteric trunk and is the second most common variation of HA (2-3\%) (14). Described cases belong to type $\mathrm{V}$ in Hiatt's classification (Table II).

The paper illustrates several types of anatomical variations of celiac trunk, hepatic artery and its main branches, based on description of two of such findings in our own clinical practice. However, in past decade there are several valuable case reports and papers (especially from liver transplant centers) which share a proper approach to the subject of the hepatic arterial variants. The first publication which mentioned CHA passing through pancreatic parenchyma comes from Michels (1951), but it still remains unclear why CHA penetrates the pancreas (it might be that CHA is developed before the fusion of dorsal and ventral pancreas) (15). Rammohan et al (8), emphasize that hepatomesenteric trunk that courses through the pancreatic parenchyma can be spared 
Table I. Uflacker's classification of CAT and its possible surgical implications.

Description

$\begin{array}{lll}\text { Classifications of CAT variations } & \text { Type } & \text { Variation }\end{array}$

Uflacker's classification $(5,11,12$

$\begin{array}{cl}\text { I } & \text { Classic coeliac trunk } \\ \text { II } & \text { Hepatosplenic trunk } \\ \text { III } & \text { Hepatogastric trunk } \\ \text { IV } & \text { Hepatosplenomesenteric trunk }\end{array}$

Frequency of $72.0-89,0 \%(5,11)$.

Most common CAT variation (3-4,4\%) (5).

IV Hepatosplenomesenteric trunk

Occurrence rate of below $1 \%$. Crucial when performing pancreatic surgery (blood supply to the duodenum come only from SMA): Accidental ligation of the SMA or branches of the common trunk can lead to the ischemia/necrosis of liver or duodenum (5).

V Gastrosplenic trunk

VI Coeliac-mesenteric trunk

During pancreatoduodenectomy (pancreatic/ peripancreatic cancers treatment): Increases a perioperative mordibity by $20-30 \%$ (5).

VII Coeliac-colic trunk

It is formed when the middle colic artery originates from coeliac trunk instead of the SMA. Difficulties and complications during transverse colon surgery: Unexpected bleeding during surgery (coeliac-colic trunk gets blood from SMA and inferior mesenteric artery) (5).

VIII No coeliac trunk No coeliac trunk can be recognised when left gastric, common hepatic and splenic arteries arise from the abdominal aorta(5).

CAT, celiac artery trunk; SMA, superior mesenteric artery.
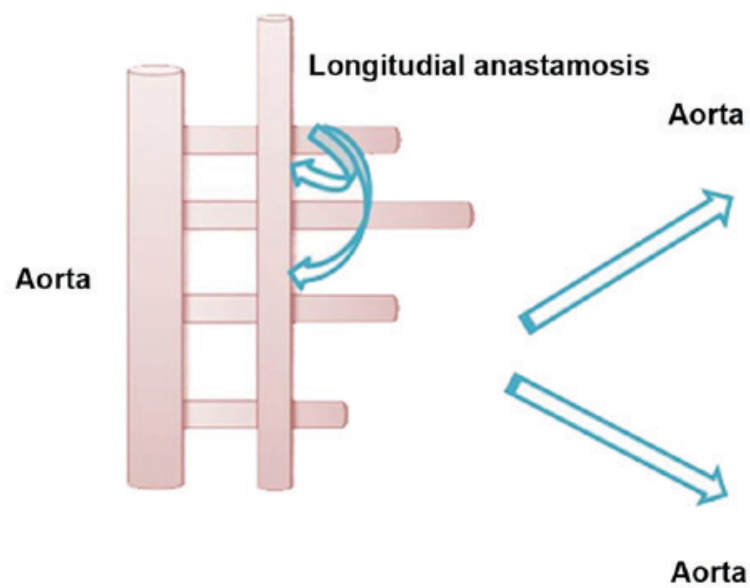

Normal anatomy

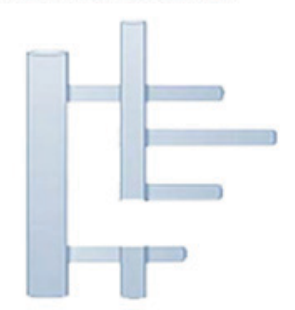

Aorta

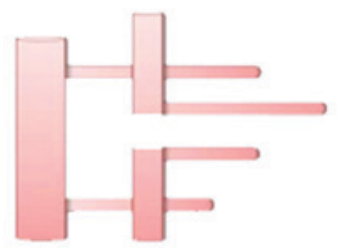

Hepatomesenteric trunk

Figure 3. Embryological development of gastrointestinal arterial supply. A schematic drawing which depicts Tandler longitudinal anastamosis and posterior regression of some of the roots resulting in normal and anomalous digestive arterial trunks.

by dividing the pancreas, but there is always a risk of not achieving tumor-free margins, what is essential in oncological surgery. If the hepatomesenteric trunk courses ventral to the pancreas, it can be displaced and dissected from the surface of the pancreas and a standard pancreaticoduodenectomy is performed. When a hepatomesenteric trunk has anastomotic connection to the LGA or another accessory artery, ligation will result in no compromise to the blood supply. In cases where the CHA is divided either inadvertently or for oncological purposes, it should be reconstructed using 
Table II. Anatomical variations of the hepatic artery: Hiatt's classification and its possible surgical implications.

\begin{tabular}{|c|c|c|c|}
\hline \multirow{2}{*}{$\begin{array}{l}\text { Classsification } \\
\text { of hepatic artery variations }\end{array}$} & \multicolumn{3}{|c|}{ Description } \\
\hline & Type & Variation & Possible surgical implications \\
\hline \multirow[t]{6}{*}{ Hiatt's classification $(9,11)$} & I & Normal anatomy & Most frequent type: $59-79,1 \%(9), 51-80 \%$ (11). \\
\hline & II & $\begin{array}{l}\text { Left hepatic artery or accessory } \\
\text { left hepatic artery relocation }\end{array}$ & $\begin{array}{l}\text { Gastrectomy should be cautiously } \\
\text { performed: Left hepatic artery emerges } \\
\text { from the left hepatic artery (ischemia } \\
\text { of the left hepatic lobe after section of } \\
\text { the left gastric artery) (9). }\end{array}$ \\
\hline & III & $\begin{array}{l}\text { Right hepatic artery or accessory } \\
\text { right hepatic artery relocation }\end{array}$ & $\begin{array}{l}\text { The most frequent described variation } \\
(7-18 \%) \text {. Procedures involving liver } \\
\text { surgery. Confusing course of the RHA: } \\
\text { After originating from SMA, the right } \\
\text { hepatic artery runs posteriorly to the } \\
\text { portal vein (9). }\end{array}$ \\
\hline & IV & $\begin{array}{l}\text { Left hepatic artery/accessory left } \\
\text { hepatic artery relocation and right } \\
\text { hepatic artery/accessory } \\
\text { right hepatic artery relocation }\end{array}$ & \\
\hline & $\mathrm{V}$ & $\begin{array}{l}\text { Common hepatic artery originating } \\
\text { from superior mesenteric artery }\end{array}$ & $\begin{array}{l}\text { Known as a hepatomesenteric trunk } \\
(2-3 \%) \text {. Altering the surgical approach } \\
\text { (interference with resection or } \\
\text { lymphadenectomy); unexpected bleeding; } \\
\text { ischemia; biliary leak; liver dysfunction (7). }\end{array}$ \\
\hline & VI & $\begin{array}{l}\text { Common hepatic artery originating } \\
\text { from the aorta }\end{array}$ & \\
\hline
\end{tabular}

SMA, superior mesenteric artery; RHA, right hepatic artery.

an autologous vascular graft such as the GDA or saphenous vein $(8,16)$. The knowledge of anatomical anomalies is of the great value during surgical interventions. The issue is crucial for, liver transplantation and resection, hepatic artery chemotherapy, gastrectomy, biliary reconstruction and especially for pancreatoduodenectomy $(17,18)$. According to Pallisera et al (14) problems related to anatomical variations of hepatic artery and coeliac axis stenosis are most prevalent arterial complications during HPB surgery. It is highlighted that intraoperative arterial complications generate longer operative time, higher transfusion rate and more postoperative complications (19).

There are some key tips which knowledge and application in clinical practice can be useful for HPB surgery to avoid unnecessary complications. First and foremost, multidetector CT with multidimensional reconstruction should be make in the preoperative management (14). Next, complete kocherisation and opening the cavity, the porta hepatis should be palpated to determine the localization of the arterial pulsation (8). Afterwards, the decision about the surgical approach and intraoperative proceeding is dependent on discovered arterial anatomical variations. The most important hepatic artery anatomical variations that the surgeon must take into consideration during pancreatoduodenectomy are: accessory RHA, accessory or displaced CHA, both arising from the SMA. If we encounter hepatic arterial anomalies the possible options for intraoperative management are ligation, dissection and traction away from the site of dissection, division and anastomosis (14). There are numerous difficulties during surgery and postoperative complications which can occur if arterial anomalies are identified: i) Partial liver ischemia and necrosis-the main problem with ligature of the displaced RHA and replaced CHA. During pancreatoduodenectomy ligation of the GDA should be delayed until the retropancreatic dissection and proper identification of the artery is complete; desirable is preoperative clamping of arteries which are going to be ligatured and post-ligature control of the blood flow $(8,14)$; ii) modification of the resection area and a risk of not achieving tumor-free margins-tough oncological compromise between safety of the procedure and radical removal of tumour (8); iii) pancreatic or biliary anastomotic leak-postoperative hepatic enzymes elevation is possible (8); and iv) unexpected bleeding-iatrogenic post- or intraoperative loss of blood (6).

In summary, we have described a two rare cases of a $\mathrm{CHA}$ originating from the SMA in combination with the topography. However, this anatomical variant is very rare (with frequency $1-3 \%$ ) should be known to surgical oncologist. In our opinion this paper is of great value for surgeons during their training period as well as the experts. Multiple arterial anomalies in a single person are found rarely. When performing operations of the pancreatic region, it is necessary to have a knowledge of anatomy including those patterns that have been rarely 
observed. The awareness of the possible extra- or intra-parenchymal path of CHA has a huge effect on decisions connected with next steps of surgery, achieving tumor-free margins, complications, patient's quality of life and costs of hospitalization. Careful review of preoperative imaging especially during multidisciplinary meeting may prevent injury to these vascular structures and later complications.

\section{References}

1. Song SY, Chung JW, Yin YH, Jae HJ, Kim HC, Jeon UB, Cho BH, So YH and Park JH: Celiac axis and common hepatic artery variations in 5002 patients: Systematic analysis with spiral CT and DSA. Radiology 255: 278-288, 2010.

2. Standring S: Gray's Anatomy: The Anatomical Basis of Clinical Practice. Elsevier, Churchill Livingstone, 2005.

3. Bhart S: Srb's Surgical Operations. Text Atlas. Jaypee Brothers Medical Pub, 2014.

4. Hiatt JR, Gabbay J and Busuttil RW: Surgical anatomy of the hepatic arteries in 1000 cases. Ann Surg 220: 50-52, 1994.

5. Michels NA: Newer anatomy of the liver and its variant blood supply and collateral circulation. Am J Surg 112: 337-347, 1966.

6. Torres K, Staśkiewicz G, Denisow M, Pietrzyk Ł, Torres A, Szukała M, Czekajska-Chehab E and Drop A: Anatomical variations of the coeliac trunk in the homogeneous Polish population, Folia Morphol (Warsz) 74: 93-99, 2015.

7. Maslarski I: Anatomical variant of the liver blood supply. Clujul Med 88: 420-423, 2015

8. Rammohan A, Sathyanesan J, Palaniappan R and Govindan M: Transpancreatic hepatomesenteric trunk complicating pancreaticoduodenectomy. JOP 14: 649-652, 2013.

9. Rela M, McCall JL, Karani J and Heaton ND: Accessory right hepatic artery arising from the left: Implications for split liver transplantation. Transplantation 66: 792-794, 1998.

10. Araujo Neto SA, de Mello Júnior CF, Franca HA, Duarte CM, Borges RF and de Magalhães AG: Multidetector computed tomography angiography of the celiac trunk and hepatic arterial system: Normal anatomy and main variants. Radiol Bras 49 : 49-52, 2016.
11. Kardile PB, Ughade JM, Ughade MN, Dhende A and Ali SS: Anomalous origin of the hepatic artery from the hepatomesenteric trunk. J Clin Diagn Res 7: 386-388, 2013

12. Ugurel MS, Battal B, Bozlar U, Nural MS, Tasar M, Ors F Saglam M and Karademir I: Anatomical variations of hepatic arterial system, coeliac trunk and renal arteries: An analysis with multidetector CT angiography. Br J Radiol 83: 661-667, 2010.

13. Uflacker R: Atlas of Vascular Anatomy: An Angiographic Approach. Lippincott Williams \& Wilkins, Baltimore, MD , 1997.

14. Pallisera A, Morales R and Ramia JM: Tricks and tips in pancreatoduodenectomy. World J Gastrointest Oncol 6: 344-350, 2014.

15. Nakamura Y, Miyaki T, Hayashi S, Iimura A and Itoh M: Three cases of the gastrosplenic and the hepatomesenteric trunks, Okajimas Folia Anat Jpn 80: 71-76, 2003.

16. Hosokawa I, Shimizu H, Nakajima M, Yoshidome $H$, Ohtsuka M, Kato A, Yoshitomi H, Furukawa K, Takeuchi D, Takayashiki T, et al: A case of pancreaticoduodenectomy for duodenal carcinoma with a replaced common hepatic artery running through the pancreatic parenchyma. Gan To Kagaku Ryoho 39: 1963-1965, 2012 (In Japanese).

17. Skórzewska M, Romanowicz T, Mielko J, Kurylcio A, Pertkiewicz J, Zymon R and Polkowski WP: Frey operation for chronic pancreatitis associated with pancreas divisum: Case report and review of the literature. Prz Gastroenterol 9: 175-178, 2014.

18. Mielko J, Kurylcio A, Skórzewska M, Ciseł B, Polkowska B, Rawicz-Pruszyński K, Sierocińska-Sawa J and Polkowski WP: Duodenal obstruction due to annular pancreas associated with carcinoma of the duodenum. Prz Gastroenterol 11: 139-142, 2016.

19. Kim AW, McCarthy WJ III, Maxhimer JB, Quiros RM, Hollinger EF, Doolas A, Millikan KW, Deziel DJ, Godellas CV and Prinz RA: Vascular complications associated with pancreaticoduodenectomy adversely affect clinical outcome. Surgery 132: 738-747, 2002.

$$
\begin{aligned}
& \text { This work is licensed under a Creative Commons } \\
& \text { Attribution-NonCommercial-NoDerivatives } 4.0 \\
& \text { International (CC BY-NC-ND 4.0) License. }
\end{aligned}
$$

